\title{
Comparison of the resistance to cyclic fatigue of One Curve, One Shape, 2Shape and EdgeFile X3 files in simulated single and S-shaped (double) curvatures
}

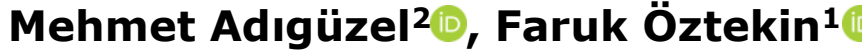 \\ ${ }^{1}$ Hatay Mustafa Kemal University, Faculty of Dentistry, Department of Endodontics, Hatay, Turkey \\ ${ }^{2}$ Fırat University, Faculty of Dentistry, Department of Endodontics, Elazığ, Turkey
}

\section{Correspondence:}

\section{Dr. Faruk ÖZTEKIN}

Firat University, Faculty of Dentistry, Department of Endodontics, Diyarbakır,

Turkey.

E-mail: foztekin@firat.edu.tr

Received: 25 November 2019 Accepted: 02 August 2020

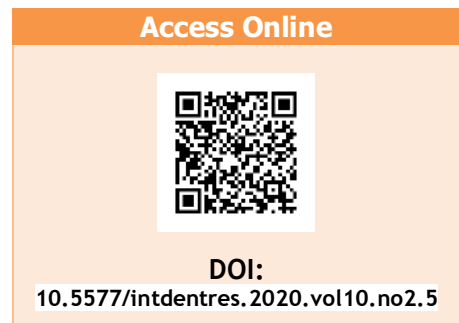

\section{Abstract}

Aim: To evaluate the fracture resistance to cyclic fatigue of One Curve (MicroMega, Besancon, Cedex, France), One Shape (Micro-Mega), 2Shape (Micro-Mega) and EdgeFile X3 (EdgeEndo, Albuquerque, NM) files in simulated root canals.

Methodology: One hundred and sixty nickel-titanium endodontic files were tested in simulated canals with single and s-shaped (double) curvatures. The One Curve (25/.06), One Shape (25/.06), 2Shape (25/.06) and EdgeFile X3 (25/.06) files were assessed ( $n=20$ for each test). All the files were rotated up to fracture occurred. The number of cycles to fracture (NCF) was determined and also, the length of the fractured file part was measured. For statistical analysis, one-way analysis of variance and Tukey HSD tests were used $(p<0.05)$.

Results: NCF for all instrument groups were significantly higher in the single curvature canal compared to the $s$-shaped curvature canal $(p<0.05)$. The EdgeFile X3 had the highest fatigue resistance in both curvature groups. The NCF of the One Curve were higher than that of the One Shape and 2Shape files ( $P<$ $0.05)$. In the s-shaped curvature canal, the files inclined to fracture more often in the apical curvature compared to the coronal curvature. There was no statistically considerable difference in the length of the fractured parts among the four experiment groups in either the single or s-shaped canal curvatures $(p>0.05)$.

Conclusion: Within the limitations of this study, fracture resistance to cyclic fatigue was the highest for the EdgeFile $X 3$ group followed by the One Curve group and lowest for the 2Shape and One Shape group.

Keywords: 2Shape, Cyclic fatigue, EdgeFile X3, One Curve, One Shape

How to cite this article: Adıgüzel M, Öztekin F. Comparison of the resistance to cyclic fatigue of One Curve, One Shape, 2Shape and EdgeFile X3 Files in simulated single and S-shaped (double) curvatures. Int Dent Res 2020;10(2):55-59.

https://doi.org/10.5577/intdentres.2020.vol10.no2.5

\section{Introduction}

Nickel-titanium (NiTi) endodontic rotary files have been mostly utilized to shape curved root canals because of their superior flexibility. However, NiTi files are probable to be subjected to fracture in clinical use. File fracture might eventuate as a result of cyclic fatigue or torsional fatigue $(1,2)$. Cyclic fatigue takes place because of repeated cycles of tension and compression, particularly when preparing curved canals, ultimately leading to fracture. Torsional fatigue takes place when tip of the file is locked to dentin walls while the shank continues to rotate. Prior studies have indicated that cyclic fatigue is the significant reason of file fracture (3). Attempts to improve better 
performing $\mathrm{NiTi}$ files require modifications in cross sections, kinematics, alloy compositions, manufacturing methods and heat treatments.

In recent years, countless innovative thermo mechanical processing and proprietary manufacturing techniques have been searched out to optimize the microstructure and the flexibility of NiTi alloys (4). For enhancement of the file properties, heat treatment has been used to reorganize the crystallographic structure of the alloy to change the transformation behavior. The innovative enhancement behind the heat treatment process is the raised Af (Austenite finish) temperature of the alloy. If "Af" is higher than the body temperature, the file will be in a mixed martensitic state, R-phase and austenitic structure. Therefore, heat treated files demonstrated a significant increase in flexibility and flexural fatigue resistance in intracanal temperature.

Recently, One Curve (Micro-Mega, Besancon, Cedex, France) and 2Shape (Micro-Mega) systems have been introduced that manufactured using heattreatment technology. One Curve instrument is a newgeneration file system using C. Wire technology. C. Wire is a proprietary process, exclusively developed for One Curve. According to manufacturer, pre-bendable, shape memory 2Shape file is made of T-Wire technology involving proprietary heat-treatment and electropolishing (5). The instrument system employs novel asymmetrical cross section with triple helix which reduces the fracture risks and improves the efficiency of brushing motion. This system use intuitive sequence with 2 shaping files in continuous rotation. One Shape (Micro-Mega) instrument is produced of traditional $\mathrm{NiTi}$ alloys, and it has an asymmetrical horizontal section design (6). EdgeFile (EdgeEndo, Albuquerque, NM, USA) systems have been presented that was produce of an annealed heat treated NiTi alloy brand named Fire-Wire. Files with different characteristics ( $\mathrm{X} 1, \mathrm{X} 3, \mathrm{X} 5, \mathrm{X} 7$ and $\mathrm{XR})$ are used in this system. Manufacturer claims that clinicians can use this system with their current system and a similar technique. EdgeFile $X 3$ files are compatible with the ProTaper and ProTaper Next (Dentsply Maillefer) rotary file system and can be employed in the same handpiece at the same speed and torque settings (7).
The objective of the present study was to evaluate the resistance to cyclic fatigue of One Curve, One Shape, 2Shape and EdgeFile X3 files. The null hypothesis tested was that there are no significant differences in the resistance to cyclic fatigue of four experiment groups.

\section{Materials and Methods}

One hundred and sixty files were evaluated in present study. The One Curve $(25 / .06)$, One Shape $(25 / .06)$, 2Shape $(25 / .06)$ and EdgeFile X3 $(25 / .06)$ files were tested in simulated canals with single and $s$ shaped curvatures $(n=20)$. Each file was examined under a stereomicroscope to describe defects before to the procedure and no files were discarded.

Cyclic fatigue testing was performed within stimulated canals with a single curvature $160^{\circ}$ curvature, $5-\mathrm{mm}$ radius) and a s-shaped curvature (coronal and apical curve is $60^{\circ}$ and $70^{\circ}$, the radius is $5 \mathrm{~mm}$ and $2 \mathrm{~mm}$, respectively, ). The stimulated canals were fabricated from a stainless steel block having single and s-shaped curvature which was especially developed and that has been used in previous studies (Fig. 1) (2, 8-10).

The insertion depth of all the files was standardized to $19 \mathrm{~mm}$. The back-and forth movements were not used. The files were employed with a motor (X-Smart; Dentsply Maillefer, Ballaigues, Switzerland) in the continuous motion as follows: One Curve at 300 $\mathrm{rpm}$, One shape at $400 \mathrm{rpm}$, 2Shape at $300 \mathrm{rpm}$, and EdgeFile $X 3$ at $300 \mathrm{rpm}$. To reduce friction, special oil was used for lubrication. For all files, the time until fracture was recorded in seconds and NCF was calculated. Furthermore, in the s-shaped curvature canals, if the fracture eventuated primarily in the apical curve, the NCF was also registered for the coronal fragment. Also, the lengths of fractured parts of files were determined by a digital caliper.

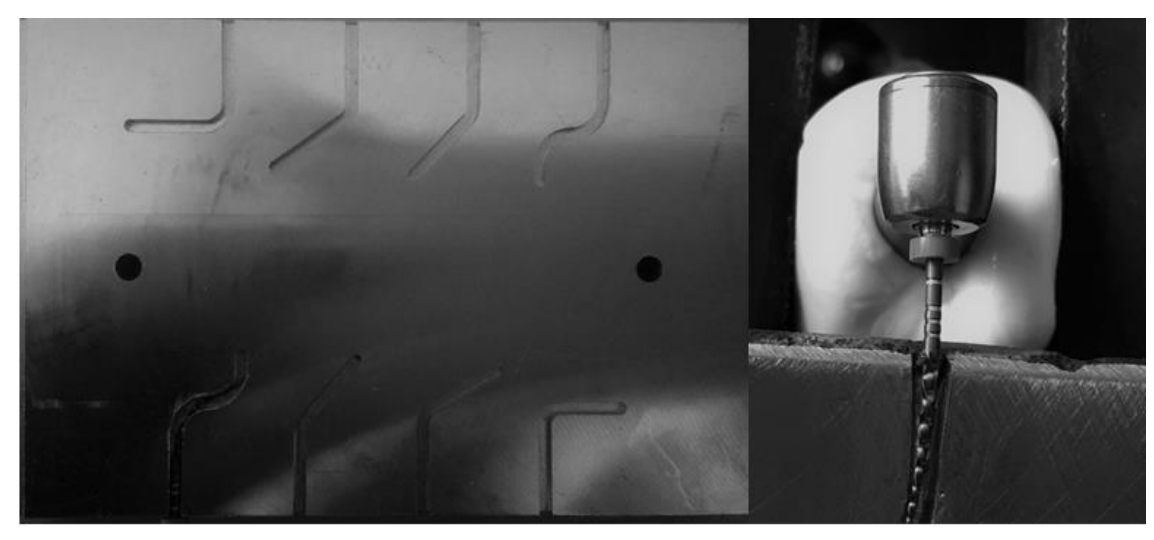

Figure 1. A photographic image of the canal metal block 


\section{Statistical Analysis}

Statistical analyses were performed with IBM SPSS V23 (SPSS Inc, Chicago, IL, USA). The Shapiro-Wilk test was used to examine the distribution of variables. A one-way analysis of variance and Tukey HSD tests were employed to determine any statistical differences among the experiment groups.

\section{Results}

The NCF for each groups are demonstrated in Table 1. NCF for all the files were significantly higher in the single curvature canal compared to the s-shaped curvature canal $(p<0.05)$. The EdgeFile $X 3$ had the highest fatigue resistance in the single and $\mathrm{s}$-shaped (coronal and apical) curvatures $(p<0.05)$. The One Curve file had higher resistance to cyclic fatigue than 2Shape and One Shape in both curvatures $(p<0.05)$. There was no significant difference in the resistance to cyclic fatigue of the 2Shape and One Shape files in either the single or s-shaped curvature. In the s-shaped curvature canal, the files inclined to fracture more often in the apical curvature compared to the coronal curvature. The fractured fragment length of the files was statistically similar among the four experiment groups in all curvatures $(p>0.05)$.

Table 1. Mean values ( \pm standard deviation) for the number of cycles to fracture (NCF) and fragment length (FL).

S-shaped Curvature
Single Curvature

\begin{tabular}{|c|c|c|c|c|c|c|}
\hline \multirow[b]{2}{*}{ Groups } & \multicolumn{2}{|c|}{ Apical curve } & \multicolumn{2}{|c|}{ Coronal Curve } & \multirow[b]{2}{*}{ NCF } & \multirow[b]{2}{*}{$\mathrm{FL}$} \\
\hline & NCF & $\mathrm{FL}$ & NCF & FL & & \\
\hline OneCurve & $1242.1 \pm 228.1 a$ & $2.18 \pm 0.26 a$ & $1409.7 \pm 257.2 \mathrm{a}$ & $5.62 \pm 0.48 a$ & $2278.9 \pm 288.1 \mathrm{a}$ & $5.58 \pm 0.28 a$ \\
\hline OneShape & $225.7 \pm 45.3 b$ & $2.28 \pm 0.24 a$ & $274.4 \pm 39.3 b$ & $5.44 \pm 0.56 a$ & $433.7 \pm 50.1 b$ & $5.49 \pm 0.35 a$ \\
\hline 2Shape & $318.4 \pm 60.7 b$ & $2.22 \pm 0.28 a$ & $382.4 \pm 63.3 b$ & $5.35 \pm 0.62 a$ & $622.9 \pm 73.8 \mathrm{~b}$ & $5.55 \pm 0.36 a$ \\
\hline EdgeEndo X3 & $2507.9 \pm 260.2 c$ & $2.24 \pm 0.22 a$ & $2709.7 \pm 400.5 c$ & $5.41 \pm 0.44 a$ & $4209.7 \pm 432.7 c$ & $5.62 \pm 0.32 a$ \\
\hline
\end{tabular}

\section{Discussion}

Many factors such as the heat treatment, crosssection, composition, and design of the endodontic $\mathrm{NiTi}$ files have considerable effects on the performance and fracture resistance of the files (11). The clinicians' knowledge on the features of endodontic files increases the rate of success in the treatments. Selecting an instrument that is more resistant to the fracture would decrease the file fractures occurring under clinical conditions (12).

The curvature angle of tooth is one of the main factors influencing the cyclic fatigue fracture of the files. Under the clinical conditions, there may be two curvatures in the same root canal. The s-shaped canals are one of the most difficult clinical settings for the use of NiTi files, and they are very hard to monitor by using conventional radiography (13). The researchers reported that the fatigue occurred very fast when the
$\mathrm{NiTi}$ files were used in s-shaped canals (14). In this study, the fracture resistance of files decreased as the curvature angle increased. The files resisted for longer periods in the curvature model having less curvature angle. The anatomy of $\mathrm{s}$-shaped-curvature canal is more difficult when compared to the single-curvature canal. The results of studies previously carried out by Pruett et al. (15) and Plotino et al. (16) corroborate the findings obtained during the present study.

In the literature, the resistances to cyclic fatigue of endodontic $\mathrm{NiTi}$ files were investigated by using several study designs. The ideal objective of the researchers was to use the cyclic fatigue tests mimicking the clinical conditions. Moreover, in this test method, it is not possible to standardize the root canal system of natural teeth and to ensure the repeatability of test circumstances for each file (8). However, the canals artificially simulated in a stainless steel block were reliably used in static and dynamic models for the analysis in this test (14). In the present study, the 
resistance to cyclic fatigue of each NiTi rotary file, which was tested by using static models since they met the standard conditions. The superiority of the static models include mostly in reducing variables, like the amplitude of axial movements (17).

In this study, the resistances to cyclic fatigue of EdgeFile X3, One Curve, 2Shape and One Shape files were compared. The results showed that the highest resistances to cyclic fatigue was found in EdgeFile X3 group, followed by the One Curve group and lowest in the 2Shape and One Shape groups. Thus, the null hypothesis that there are no differences in the resistance to cyclic fatigue of the groups was rejected.

Topçuoğulları et al. (18) compared the resistances to cyclic fatigue of One Curve, EdgeFile, HyFlex CM (Coltene-Whaledent, Switzerland) and ProTaper Next (Dentsply Maillefer, Switzerland) file systems at two different temperatures and they reported that there was no significant difference in cycling fatigue resistance between One Curve and EdgeFile groups at both temperatures. However, Khalil (19) tested the effect of heat treatment technique on the resistance to cyclic fatigue for OneCurve and EdgeFile and reported that the EdgeFile NiTi rotary files had high flexibility and high resistant to cyclic fatigue than OneCurve did. It was thought that the difference in the results of these two researchers is due to the variables in dynamic mode used in both studies. In this study, both files exhibited long time to fracture, however, the highest resistance to cyclic fatigue was found in EdgeFile group. According to the manufacturer, the annealed heat-treated Firewire $\mathrm{NiTi}$ provides significantly flexibility. Heat treatment process as cryogenically tempered may have increased resistance to cyclic fatigue of EdgeFile X3 files. A another factor could contribute to resistance to cyclic fatigue; different cross sections of OneCurve influence fracture resistance as a weakening factor (19).

Elnaghy ve Elsaka (20) examined in simulated canals with 90-degree angle of curvature the resistances to cyclic fatigue of One Curve, 2Shape, ProFile Vortex (Dentsply Tulsa Dental, Tulsa, OK) and One Shape NiTi rotary files and revealed that One Curve files had statistically significantly higher resistance to cyclic fatigue than 2 Shape and One Shape files did. In the present study, 2Shape files had fracture resistance lower than One Curve and EdgeFile files and higher (even not significant) than One Shape files. 2Shape files are produced from T-Wire heat-treated technology that might improve the fatigue resistance. In another study, Staffoli et al. (21) revealed that One Curve files are significantly more resistant to cyclic fatigue than One Shape files at all temperatures tested. These studies corroborate the results of the present study. It is thought that the difference between the files having the same design (One Curve \& One shape) originates from the $\mathrm{C}$. Wire used in the production of One Curve files. C. Wire technology utilizes a proprietary heat treatment with a controlled memory characteristic that advanced the resistance to cyclic fatigue of the instrument (22). Besides the alloy used in the file production, the heat treatments also have effect on the resistance to cyclic fatigue of the files.

\section{Conclusions}

Within the limitations of present study, resistance to cyclic fatigue was the highest for the EdgeFile X3 group followed by the One Curve group and lowest for the 2Shape and One Shape group.

Peer-review: Externally peer-reviewed.

Author Contributions: Conception - M.A., F.Ö.; Design - M.A., F.Ö.; Supervision - M.A.; Materials - M.A., F.Ö.; Data Collection and/or Processing - F.Ö.; Analysis and/or Interpretation - M.A.; Literature Review - M.A., F.Ö.; Writer - M.A.; Critical Review - F.Ö.

Conflict of Interest: No conflict of interest was declared by the authors.

Financial Disclosure: The authors declared that this study has received no financial support.

Acknowledgements: The author would like to thank Micromega and EdgeEndo for donating the files used in this study. The author declares that this study has received no financial support. No conflict of interest was declared by the author.

\section{References}

1. Adiguzel M, Capar ID. Comparison of cyclic fatigue resistance of WaveOne and WaveOne Gold Small, Primary, and Large Instruments. J Endod 2017;43(4):623-7. (Crossref)

2. Adiguzel M, Turgay B. Comparison of the cyclic fatigue resistance of reciproc and reciproc blue nickel-titanium instruments in artificial canals with single and double (sshaped) curvatures. Eur Endod J 2017;2(1):32-32 (Crossref)

3. Topcuoglu HS, Topcuoglu G. Cyclic fatigue resistance of Reciproc Blue and Reciproc Files in an s-shaped canal. J Endod 2017;43(10):1679-82. (Crossref)

4. Gutmann JL, Gao Y. Alteration in the inherent metallic and surface properties of nickel-titanium root canal instruments to enhance performance, durability and safety: a focused review. Int Endod J 2012;45(2):113-28. (Crossref)

5. Ozyurek T, Gundogar M, Uslu G, Yilmaz K, Staffoli S, Nm G, et al. Cyclic fatigue resistances of Hyflex EDM, WaveOne gold, Reciproc blue and 2shape NiTi rotary files in different artificial canals. Odontology 2018;106(4):408-13. (Crossref)

6. Gundogar M, Ozyurek T. Cyclic Fatigue Resistance of OneShape, HyFlex EDM, WaveOne Gold, and Reciproc Blue Nickel-titanium Instruments. J Endod 2017;43(7):1192-6. (Crossref)

7. Versiani MA, Carvalho KKT, Mazzi-Chaves JF, Sousa-Neto MD. Micro-computed Tomographic Evaluation of the Shaping Ability of XP-endo Shaper, iRaCe, and EdgeFile Systems in Long Ovalshaped Canals. J Endod 2018;44(3):489-95. (Crossref)

8. Topcuoglu HS, Duzgun S, Akti A, Topcuoglu G. Laboratory comparison of cyclic fatigue resistance of WaveOne Gold, Reciproc and WaveOne files in canals with a double curvature. Int Endod J 2017;50(7):713-7. (Crossref)

9. Al-Sudani D, Grande NM, Plotino G, Pompa G, Di Carlo S, Testarelli L, et al. Cyclic fatigue of nickel-titanium rotary instruments in a double (S-shaped) simulated curvature. J Endod 2012;38(7):987-9. (Crossref) 
10. Larsen CM, Watanabe I, Glickman GN, He J. Cyclic fatigue analysis of a new generation of nickel titanium rotary instruments. J Endod 2009;35(3):401-3. (Crossref)

11. Elsaka SE, Elnaghy AM, Badr AE. Torsional and bending resistance of WaveOne Gold, Reciproc and Twisted File Adaptive instruments. Int Endod J 2017;50(11):1077-83. (Crossref)

12. Gao Y, Gutmann JL, Wilkinson K, Maxwell R, Ammon D. Evaluation of the impact of raw materials on the fatigue and mechanical properties of ProFile Vortex rotary instruments. J Endod 2012;38(3):398-401. (Crossref)

13. Topcuoglu HS, Topcuoglu G, Akti A, Duzgun S. In vitro comparison of cyclic fatigue resistance of ProTaper Next, HyFlex CM, OneShape, and ProTaper Universal Instruments in a canal with a double curvature. J Endod 2016;42(6):969-71. (Crossref)

14. Capar ID, Kaval ME, Ertas H, Sen BH. Comparison of the cyclic fatigue resistance of 5 different rotary pathfinding instruments made of conventional nickel-titanium wire, $M$-wire, and controlled memory wire. J Endod 2015;41(4):535-8. (Crossref)

15. Pruett JP, Clement DJ, Carnes DL, Jr. Cyclic fatigue testing of nickel-titanium endodontic instruments. J Endod 1997;23(2):77-85.

16. Plotino G, Grande NM, Melo MC, Bahia MG, Testarelli L, Gambarini G. Cyclic fatigue of NiTi rotary instruments in a simulated apical abrupt curvature. Int Endod J 2010;43(3):22630. (Crossref)
17. Bueno CRE, Cury MTS, Vasques AMV, Sivieri-Araujo G, Jacinto RC, Gomes-Filho JE, et al. Cyclic fatigue resistance of novel Genius and Edgefile nickel-titanium reciprocating instruments. Braz Oral Res. 2019;33:e028. (Crossref)

18. Topcuoglu HS, Topcuoglu G, Kafdag O, Balkaya H. Effect of two different temperatures on resistance to cyclic fatigue of one Curve, EdgeFile, HyFlex CM and ProTaper next files. Aust Endod J 2020;46:68-72 (Crossref)

19. Khalil WA. Effect of heat treatment on cyclic fatigue, flexibility and phase transformation of two controlled memory niti rotary instruments. Egypt. Dent. J 2019;65(2):1463-8. (Crossref)

20. Elnaghy AM, Elsaka SE. Cyclic fatigue and torsional resistance of heat-treated One Curve compared with different nickeltitanium rotary instruments. Endodontic Practice Today 2019;13(3).

21. Staffoli S, Grande NM, Plotino G, Ozyurek T, Gundogar M, Fortunato $L$, et al. Influence of environmental temperature, heat-treatment and design on the cyclic fatigue resistance of three generations of a single-file nickel-titanium rotary instrument. Odontology 2019;107(3):301-7. (Crossref)

22. Elnaghy AM, Elsaka SE. Cyclic fatigue resistance of One Curve, 2Shape, ProFile Vortex, Vortex Blue, and RaCe nickel-titanium rotary instruments in single and double curvature canals. J Endod 2018;44(11):1725-30. (Crossref) 